\title{
Reducing the soil structure destruction along the rut and increasing the traction properties of the tractor by using the rear grouser of the track link
}

\author{
V.N. Kozhanov ${ }^{1, *}$, M.A. Rusanov ${ }^{1,2}, M . G$. Shtyka $^{2}$, and V.S. Kukhar ${ }^{3}$ \\ ${ }^{1}$ FSBEI HE South Ural State Agrarian University, Chelyabinsk, Russia \\ ${ }^{2}$ FSAEI HE South Ural State University (National Research University), branch in the Miass, Russia \\ ${ }^{3}$ FSBEI HE Ural State Agrarian University, Yekaterinburg, Russia
}

\begin{abstract}
The traditionally used mixed grouser of the metal track link causes a decrease in the traction qualities of the agricultural tractor. The use of a rear grouser on the track link, in our opinion, will significantly improve the traction properties of an agricultural tractor with a metal track and reduce the soil destruction. When the rear grouser is immersed in the soil, an additional horizontal deformation of the soil occurs, which changes the law of horizontal deformation distribution along the support surface of the trackdrive, which ensures the alignment of the link shares in the implementation of the tangential traction force. This leads not only to a reduction in the trackdrive skidding, but also to a reduction in tractor rolling losses. Comparative tests of the T-4A tractor with a serial track, and a track on which links the front grousers were removed showed that the maximum traction power increases from 59 to $65 \mathrm{~kW}$, the skidding with a hook load of $40 \mathrm{kN}$ decreases from 14.6 to $9.4 \%$, the rolling resistance coefficient decreases from 0.093 to 0.072 , eliminates the "scissors" effect, which will reduce the number of erosive-dangerous particles in the track trace to $30 \ldots 40 \%$, which is $5.6 \ldots 4.25$ times less than in agricultural tractors with a mixed grouser, which confirms the effectiveness of their use.
\end{abstract}

\section{Introduction}

One of the most promising ways to increase the productivity of machine-tractor units is to increase the traction force of the track tractor, with the same adhesion weight, by improving the trackdrive. Obtaining the necessary traction force by increasing the tractor's adhesion weight at increased processing speeds leads to a sharp increase in the mechanical impact on the soil. There is a decrease in the yield of agricultural crops due to the intensive destruction of the structure and over-compaction of the soil $[1,2]$. Therefore, increasing the traction force of agricultural track tractors, it is impossible not to consider the features of their work, which consists in the fact that the supporting surface of their track assembly is a complex biological environment that has an invaluable property - fertility [2]. Since the

\footnotetext{
* Corresponding author: kozhanoww@mail.ru
} 
beginning of the production of the first agricultural caterpillat tractors and up to the present time, all metal tracked contours of agricultural tractors of the RF have traditionally used the combined position of the grousers on the track link. On each link, the disjoined grousers are located at the front and rear eyes and are respectively called front and rear. The rear and front grousers are determined by the direction of tractor movement for the link lying on the support surface. After assembling such links into a track chain, each joint contains grousers from two adjacent links Fig. 1. The front grousers of one link and the rear grousers of the other in each joint form a common grousers, which is called a combined one. The authors [2] consider this link design to be optimal, in terms of reducing the clogging of the running system with soil and snow p. 71, as well as ensuring stability from lateral sliding when the tractor moves on the slopes p.74. This article substantiates the position of the grouser on track link in terms of improving the traction-caupling qualities of the tractor and reducing the harmful effects of the trackdrive on the soil.

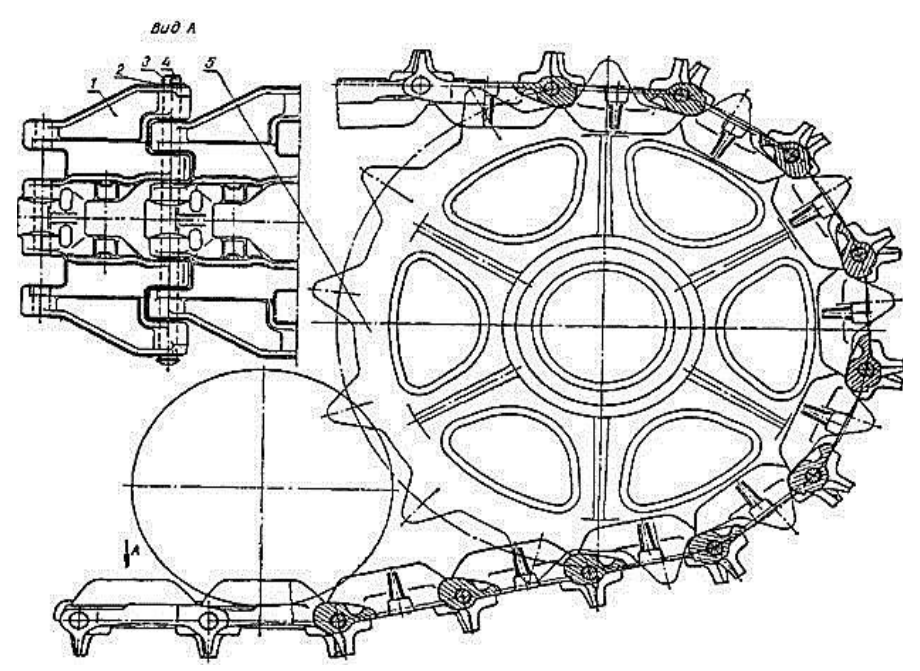

Fig. 1. Tractor caterpillar with mixed grouser. 1 - track link; 2 - washer; 3 - pin; 4 - cotter pin; 5 driving wheel.

\section{Theoretical research}

At present, in tractor theory, when considering the coupling of the trackdrive with the soil, the influence of the location of the grousers on the link of the track is not sufficiently evaluated [1]. Even Professor E.D. Lvov, in 1946, rightly noted the most important role of the spur (grouser) of the track link in the formation of tractor traction qualities "... with the rear location of the spurs, you can expect less losses from the skidding of the track and less destruction of the soil, that is, reducing the energy consumption for the spurs" [3] p. 113.

The trajectory of the grousers when they are immersed in the soil and when they leave the soil depends on their location along the length of the track links are considered in sufficient detail in our works $[1,4,7]$.

So, Figure 2 shows the limit options for the location of the grouser - front and rear. The trajectories of any grouser points when they are immersed in the soil are circles which center coincides with the center of the rear joint of the link in question, if to neglect the track chain skidding.

The trajectories of any grouser points at their exit from the soil, with the assumed absence of track skidding, are circles which center coincides with the center of the front joint of the link in question. 


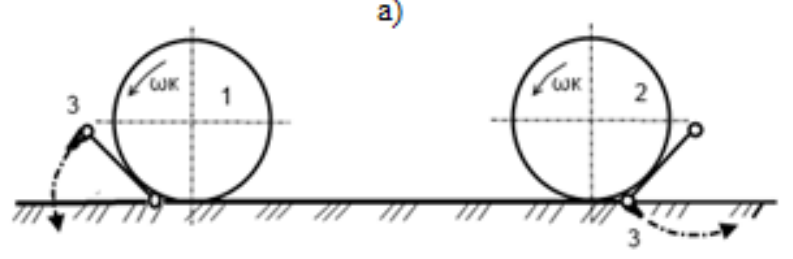

b)

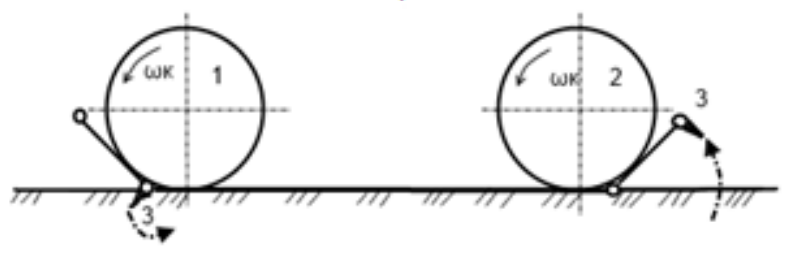

Fig. 2. Diagram of the interaction of the support branch of the trackdrive with the soil. a - with the front grouser; $b$ - with the rear grouser; 1 - front support roller; 2 - rear support roller; 3 - grouser; $\omega_{\mathrm{k}}$ - angular rate.

In the front position (Fig. 2a), the grouser dips into the soil relatively easily, since any point of the grouser moves along the circumference of the largest possible radius, approximately equal to the length of the track chain link [4]. The exit of the front grouser begins with its rotation in the buried state, then the movement takes place in an arc of small radius, the center of which is located on the axis of the front joint. At the same time, the grouser, turning in the compacted soil, destroys it, and at high speed of the tractor throws the chipped and compacted volume of soil into the engagement zone of the traction wheel, on the arc and free branch of the track.

With further movement and fluctuations of the free track branch, the soil is sifted onto the treadmill of the support branch, where there is an intensive destruction of its structure, which leads to an increase in the number of erosion-hazardous particles on the tractor trace, reduces the trackdrive efficiency. At high operating speeds and low humidity, chipped soil is thrown out from under the driving wheel, increasing the dustiness on the tractor trace, forming a dust cloud.

The rear position of the grouser (Fig. 2b) causes some compaction of the soil when the grouser is immersed in the soil [1]. This compaction of the soil should be considered desirable, since it will increase the reliability of the grouser stop in the horizontal direction, i.e. reduce the track skidding loss. The release of the grouser from the soil is relatively easy, since any of its points moves circumferentially with the largest possible radius, approximately equal to the length of the track chain link.

The combined grousers of adjacent links in the joint act on the soil like "scissors" [4], destroying soil structure. Moreover, in each joint of the most common seven-eye track, the grousers form six (Fig. 1 "view A") "scissors". The mutual rotation of adjacent links, which combined grousers on agricultural backgrounds are usually completely embedded in the soil, causes the soil structural components to be grinded and its diffusion. The soil adhesion under the track is deteriorated, its resistance to shear by the grouser decreases, leading to an increase in skidding, a decrease in the forward speed, and, consequently, the productivity of the tractor unit. The destruction of the soil structure consumes energy, which causes increased tractor rolling resistance and additional fuel consumption.

When the link is laid by the front roller, the transition of each roller through the track joints and the exit of the link from under the rear roller, the relative rotation of the adjacent links and hooks occurs at an angle $\alpha=\alpha_{1}+\alpha_{2}+\alpha_{3}$ (Fig. 3) [4]. 


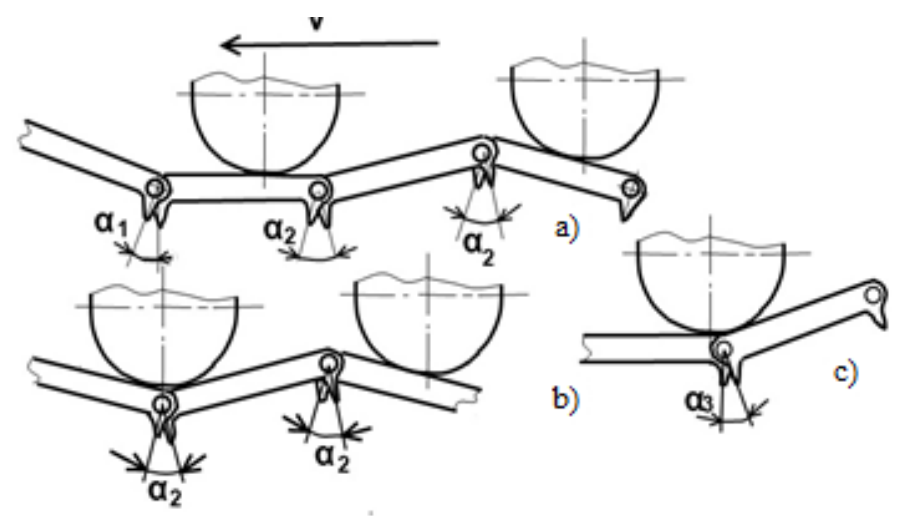

Fig. 3. Relative rotation of the grousers of adjacent links. a - when laying the link with the front roller; $\mathrm{b}$ - on the support branch; $\mathrm{c}$ - when the link leaves the rear roller.

The destruction of the soil structure due to the impact of the trackdrive on it is estimated by the total angle of rotation $\sum \alpha$, made by two adjacent links when they pass along the entire length of the support surface. $\sum \alpha$ affects the amount of erosion-hazardous particles in the soil. The regularities of changes in the number of erosive-hazardous particles along the trace depending on the total angle of rotation of the combined links were studied by Shatalov V.T. [5]. For the DT-75 tractor with an elastic suspension of the support rollers $\sum \alpha=90 \ldots 115^{\circ}$, while the number of erosion-hazardous particles on the track trace increases by $170 \%$, and with a double pass by $240 \%$, which is significantly higher than for tractors, the track link of which has one grouser.

The process of soil deformation by grousers of the tracks in the direction opposite to the tractor movement is described in detail in $[6,7]$, and occurs up to the complete release of the grouser of the last rear link of the support branch of the track contour.

In this process, the grousers that are currently engaged with the soil additionally compress the soil by the value $\Delta_{\mathrm{i}}$, which depends on the trackdrive skidding [1]. All grousers shift and cut the soil by the same amount $\Delta_{\mathrm{i}}$, the first by $\Delta_{\mathrm{i}}$, the second $\Delta_{\mathrm{i}}+\Delta_{\mathrm{i}}=2 \Delta_{\mathrm{i}}$, the third $\Delta_{\mathrm{i}}+\Delta_{\mathrm{i}}+\Delta_{\mathrm{i}}=3 \cdot \Delta_{\mathrm{i}}$ etc.

When the tractor is moving steadily, the soil shift increases on each subsequent grouse and reaches the maximum value of $\Delta_{\max }=\delta \cdot L$ on the last one (here $\delta$ is the skid of the trackdrive, $L$ is the length of the support branch of the track contour) or $\Delta_{\max }=\mathrm{n} \cdot \Delta_{\mathrm{i}}$, where $\mathrm{n}$ is the number of grousers in the engagement of the support branch of the trackdrive.

Thus, when the trackdrive works with the front grousers with the length of the support surface $L=1.5 \ldots 2.5 \mathrm{~m}$ at $\delta=10 \%$, the deformation value can vary from zero for the first link to $0.15 \ldots 0.25 \mathrm{~m}$ for the last one [1].

The sinking of the rear grouser, at the beginning, occurs in the same way as the front one, $\gamma$ but at an angle until the adjacent link is completely laid. Then, at the moment of support roller or guide wheel underrun on the link of the rear grouser, it rotates relative to the pin axis. The rotation of the rear grouser in the merged state causes an additional horizontal deformation of the soil by the value $\Delta S$ (Fig. 4), which will be determined by the formula [7]: 


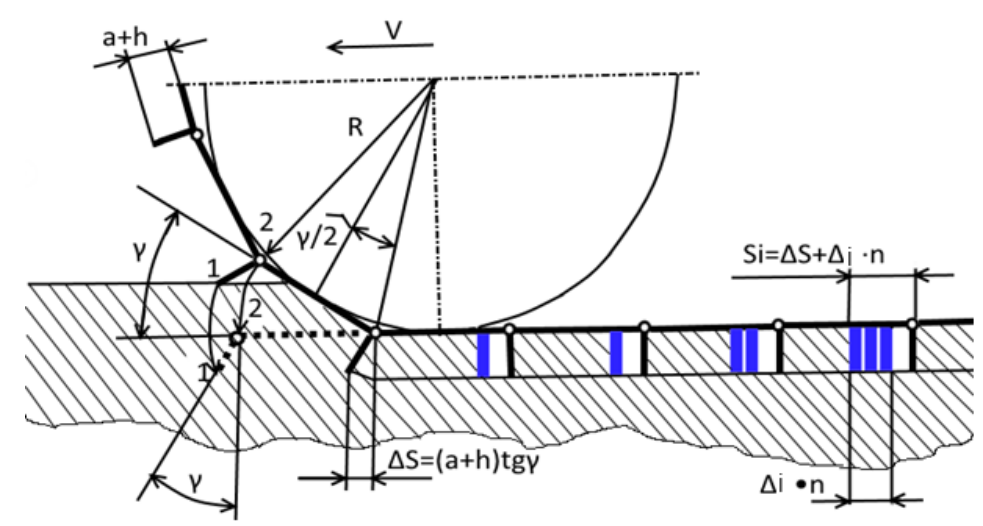

Fig. 4. The scheme of laying a link with a rear grouser in the soil with a guide wheel and the formation of a preliminary soil pre-pressing.

$$
\Delta S=\left(\mathrm{a}+h_{\mathrm{g}}\right) \operatorname{tg} \gamma
$$

where $\gamma$ - angle of rotation of the rear grouser, equal to the angle of inclination of the lower front branch of the track contour;

$h_{\mathrm{g}}$ - grouser height;

a - coefficient determined by the link design.

$$
a=\frac{d}{2}+c,
$$

where $d-$ is the diameter of the track link joint;

$c$ - link thickness.

Then the maximum horizontal total soil deformation of the last link will be equal to

$$
\Delta_{\max }=\Delta S+(\delta \cdot L)
$$

To determine the tangential traction force of trackdrive, it is necessary to have a dependence of the shear stresses that occur in the soil when the trackdrive acts on it on the horizontal deformations $\Delta_{\max }[8]$. There are quite a few known dependences between shear stress and deformation, each of which has its own advantages and disadvantages. One of the most successful one is the dependence proposed by Professor V.V. Katsygin in work [8], which shows its great functionality. Foreign researchers widely use the dependence proposed by M.G. Becker [9]. The dependencies described above are widely used to describe the interaction of trackdrives with the soil $[10,11,12,13]$. The considered dependences assume to consider the problem as a flat one, while the soil deformation of each of the areas of the trackdrive support surface is considered independently of each other; deformations in the transverse direction, soil heating by the lateral surface, etc. are not taken into account [16].

At present, the finite element method is widely used for the study of the stress-strain state of soils. For example, in works $[14,15,16]$, the DEM model describing the soil as a set of interacting particles was used to study the interaction of the track with the soil. In work [16], the finite element method made it possible to perform calculations of volumetric models of a high-speed vehicle track and a soil mass using the LS-DYNA software package [17], but the process of laying the track on the soil under the first support roller is not considered. To test the main characteristics of the soils in work [16], the experimental 
dependences of the normal force on the depth of immersion are also compared, obtained by the formula of V.V. Katsygin, are also compared as well as the corresponding dependences obtained from the numerical experiment by the finite element method for the considered soils. The analysis of the presented results confirms their compliance, which indicates sufficient accuracy of the calculations [16].

Next, to derive the tangential thrust force of trackdrive with rear grouser, we will use one of the well-known methods proposed by V.V. Guskov [18] p.130...131. His method of calculation is based on the formula of V.V. Katsigin which well approximates the dependence of the shear stress of the soil on its horizontal deformation expression 1.6 p. 19 [18]. Given that, the projection of the resultant reaction of the soil on the plane coinciding with the surface of the trackdrive, the tangential thrust force $F_{K}$ is equal to the sum of the tangential reactions of the soil resulting from the shear and soil brick cut, i.e.

$$
\mathrm{F}_{\mathrm{K}}=\mathrm{F}_{\mathrm{K} . \mathrm{SD}}+\mathrm{F}_{\mathrm{K} . \mathrm{sr}}
$$

Let's calculate the soil reactions that occur due to the shear of the soil bricks. The total soil deformation from the rear grousers, which is equal at the beginning of the engagement $\Delta S$ and reaches at the end $\Delta_{\max }=\Delta S+(\delta \cdot L)$, can be represented as a trapezium (Fig. 5). Then the soil shear deformation by the rear grouser located at a distance $x$ from the beginning of the engagement is equal to $\Delta S+\delta x$ [7]. Let's select an elementary section of the track $d x$ on the support branch. The tangential thrust force realized in this section has the form

$$
d F_{K . S D}=\tau_{S D} D a
$$

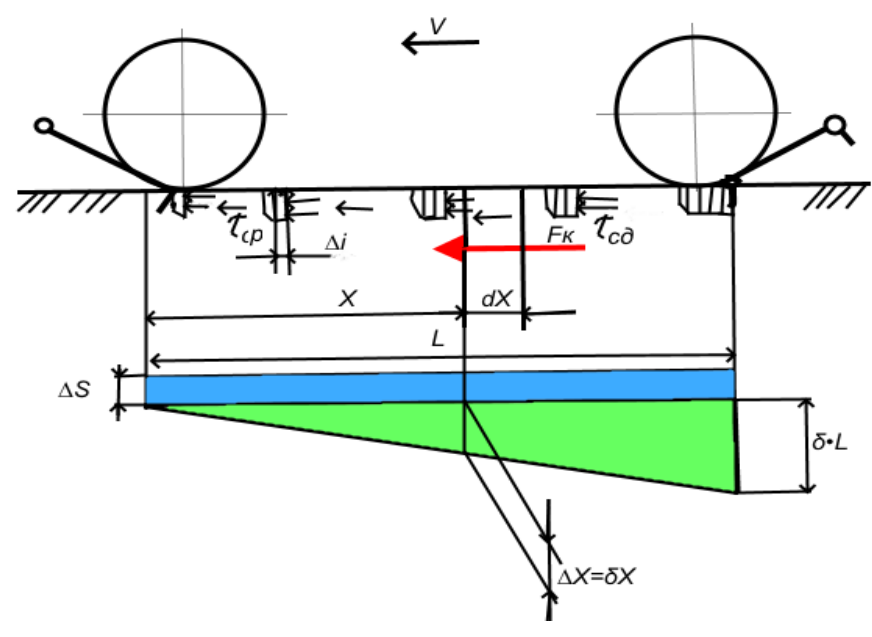

Fig. 5. Epure of the shear strain distribution.

where $d A$ is the elementary platform of the track track support surface, equal to $d A=b d x, b$ is the width of the grouser of the track link; dx is the length of the elementary platform.

Tangential traction force of the track support branches required to overcome the shear reaction of soil bricks,

$$
F_{K . S D}=\int_{0}^{L} \tau_{S D} d A,
$$

where $L$ is the length of the trackdrive support surface. In general, it depends on the normal pressure $p_{x}$ of the soil deformation, its physical and mechanical properties, and the 
parameters of the trackdrive [18]. Of these, the variables are normal pressure and shear strain, i.e.

$$
\tau_{S D}=f\left(p_{x,}, \Delta_{x}\right)
$$

Substituting the expression of V.V. Katsigin p. 19 [18] in the formula (2), we obtain the tangential traction force of the supporting branches of two tractor tracks, which is necessary for soil bricks cut.

$$
F_{K . S D}=2 \cdot \int_{0}^{L} \tau_{S D} d A=2 \int_{0}^{L} b \cdot f_{S K} \cdot p_{x}\left(1+\frac{f_{p r}}{c h \frac{\delta x}{k_{\tau}}}\right) \cdot t h \frac{\delta x}{k_{\tau}} d x
$$

where $f_{S K}$ - the coefficient of sliding friction; $p_{x}$ - the normal pressure of the trackdrive on the soil; $f_{p r}$ - the reduced coefficient of friction, for soils of normal humidity, it can be calculated by the empirical formula [18] p. 19 .

$$
f_{S K}=2,55\left(\frac{f_{p}-f_{S K}}{f_{S K}}\right)^{0,825}
$$

$f_{p}$ - coefficient of static friction.

$k_{\tau}$ - coefficient of surface deformations, $\mathrm{m}$;

$k_{\tau}$ it is proportional to the critical strain $\Delta_{0}$ (at which the shear stress has the maximum value) and depends on the reduced friction coefficient $f_{p r}$

$$
k_{\tau}=\frac{\Delta_{0}}{\operatorname{arcch}\left(\frac{1+\sqrt{1+f_{p r}^{2}}}{f_{s k}}\right)}
$$

C with sufficient accuracy for mineral soils of normal humidity, $k_{\tau}$ can be calculated by the formula $k_{\tau}=0,4 \cdot t[18]$ p. 19 .

ch - hyperbolic cosine; th - hyperbolic tangent,

arcch - hyperbolic arctangent.

Under the accepted assumption of a uniform pressure distribution along the length of the track support branch [18], i.e. when $p_{x}=p_{s r}=G /(2 b L)$ (here $G$ is the tractor gravity force), the tangential tractive force of the soil bricks

$$
F_{K . S D}=2 \int_{0}^{L} \frac{f_{S k} \cdot G}{L} \cdot\left(1+\frac{f_{p r}}{\operatorname{ch} \frac{\delta x}{k_{\tau}}}\right) \cdot \operatorname{th} \frac{\delta x}{k_{\tau}} d x=\frac{f_{S k} \cdot G \cdot k_{\tau}}{\Delta S+(\delta \cdot L)}\left[\ln \operatorname{ch} \frac{\Delta S+(\delta \cdot L)}{k_{\tau}}-f_{p r}\left(\frac{1}{c h \frac{\Delta S+(\delta \cdot L)}{k_{\tau}}}-1\right)\right]
$$

Tangential tractive force required to overcome the reactions of the two trackdrives' cut p. 131 [18],

$$
F_{K . S R}=4 \tau_{s r} h_{\mathrm{g}} \frac{L}{t_{g}},
$$

where $\tau_{s r}$ - the cut stress, Mpa; $h_{\mathrm{g}}-$ the grouser height, $\mathrm{m}$.

Thus, we obtain an expression for determining the total tangential traction force of the trackdrive with a rear-mounted grouser.

$$
F_{K}=\frac{f_{S k} \cdot G \cdot k_{\tau}}{\Delta S+(\delta \cdot L)}\left[\ln c h \frac{\Delta S+(\delta \cdot L)}{k_{\tau}}-f_{p r}\left(\frac{1}{\operatorname{ch} \frac{\Delta S+(\delta \cdot L)}{k_{\tau}}}-1\right)\right]+4 \tau_{s r} h_{\mathrm{g}} \frac{L}{t_{\Gamma}}
$$


Let us analyze the dependence of the tangential force $F_{K}$ on the coefficient of trackdrive skidding with the front and rear grousers. To do this, in Figure 6 we will match the calculated data for the front grouser p. 132 [18], with our data, obtained for the rear grouser. The initial data for the calculation will be the same. The coefficients of static friction $f_{p}$ and sliding $f_{S K}$ are determined depending on the trackdrive pressure on the soil (see Fig. 1.5.) [18] p. 16.

The initial data for the calculations are taken from [18]:

$\mathrm{A}=$ const $=1,32 \mathrm{~m}^{2} ; L=1,68 \mathrm{~m} ; t_{g}=0,18 \mathrm{~m} ; h_{\mathrm{g}}=0,05 \mathrm{~m} ; k_{\tau}=0,08 \mathrm{~m} ; \tau_{s r}=1,7 \mathrm{kPA}$;

$d=0,02 \mathrm{~m} ; c=0,02 \mathrm{~m} ; \gamma=10,5 \ldots .25^{\circ}$ angle of rotation of the rear grouser, equal to the angle of inclination of the lower front branch of the track contour.

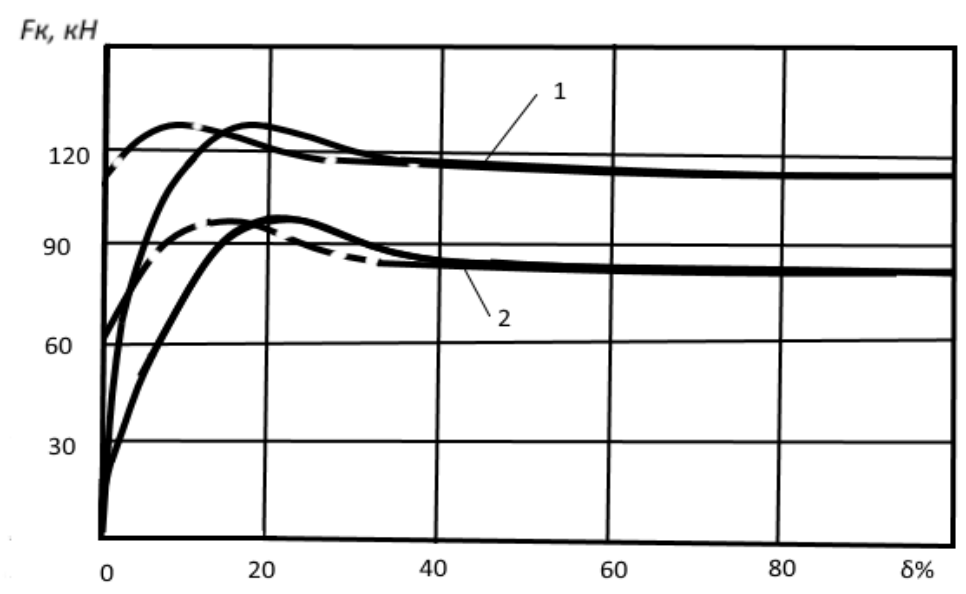

Fig. 6. The dependence of the tangential traction force of the trackdrive with a different location of the grouser on the skidding coefficient on the light loam stubble, with different tractor weight: $1-\mathrm{G}$ $=150 \mathrm{kN} ; 2-G=110 \mathrm{kN} ; \gamma=10,5^{\circ} ;-$ - front, - - - rear grouser.

Fig. 6 shows that the tangential traction force increases until the skidding coefficient reaches a certain maximum value, after which it begins to decrease. The decrease in the tangential traction force with a large skidding is explained by the fact that the soil bricks are cut off, pinched between the soil grousers and mixed together with the track. Since the values of the sliding shear stress are less than the maximum (resting stresses), therefore, the tangential traction force begins to decrease. The skidding, at which the maximum tangential traction force develops, for the trackdrive with front grouser is in the range of $18-20 \%$, the results obtained are in good agreement with the data [18] p.132. However, it should be noted that in the track with a rear position of the grouser, due to additional soil prepressing, the maximum tangential traction force occurs at lower skidding values by $6 \ldots 9 \%$.

\section{Methods of experimental research}

To assess the effectiveness of the use of the rear grouser, as well as $\sum \alpha$ value of trackdrive, experimental studies were conducted. The object of research was a T-4A tractor with an A$01 \mathrm{M}$ engine. During the research, the tractor was equipped with two variants of tracks:

- serial with a combined grouser;

- experimental with a disjoined rear grouser.

The experimental version was obtained from the serial track, by mechanical removal of part of the combined grouser Fig. 7. The total grouser surface area, in the experimental 
version, was almost two times less than in the serial version. To ensure comparability of the results for the two track link designs, the tests were carried out on the same day and in the shortest possible time.

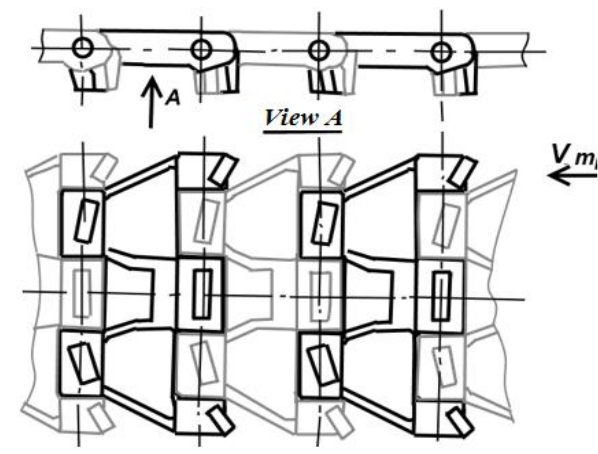

serial

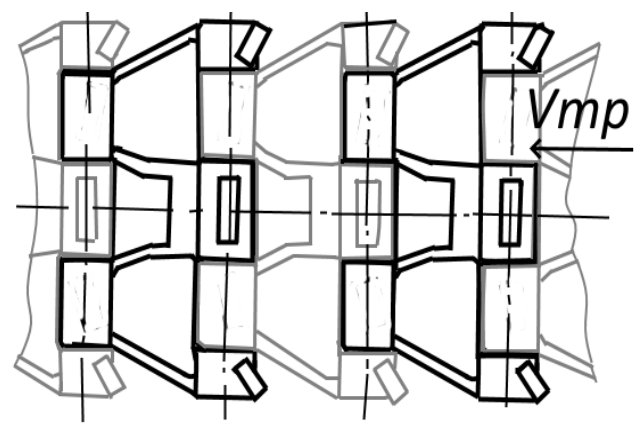

experimental

Fig. 7. Variations of trackdrives. Vtr- direction of the tractor speed.

The angle of rotation of the adjacent links was measured by angular displacement sensors, the main element of which was a variable resistor with a linear characteristic, included in the scheme of an equal-arm bridge through compensation resistances. The housing of the variable resistor was fixed to the link so that the axis of the eye of the track link coincided with the rotation axis of the movable resistor contact. The movable contact is connected to the adjacent link through a system of rods with gap compensators. Thus, when turning any of the two adjacent links, the movable contact will rotate relative to its housing. Turning the movable contact causes a change in the resistance of one of the half-bridges, causing an out-of-balance current. The value of the out-of-balance current will be proportional to the relative angle of link rotation $\alpha$.

In other respects, the method of comparative tests met the requirements of GOST 70572001 "Agricultural tractors. Test methods". The tests were carried out on a plowed field, with the grouse completely submerged in the soil. Loading of the tractor was carried out by the dynamometer laboratory DL-10, cultivator subsurface plow KPG-2-150.

\section{Results of experimental research}

The results of the comparative traction tests are shown in Figure 8. The large value of the trackdrive skidding at a nominal traction force of $22.6 \%$ and $14.4 \%$ for serial and experimental tracks is explained by the high soil moisture, which in both cases was $25 \%$.

At a hook load of $40 \mathrm{kN}$, the skid rate was $14.9 \%$ for the serial track and $9.6 \%$ for the experimental track.

The maximum hook power of the tractor on V, VI and VII gears on the experimental track is $64.5 ; 65.0 ; 64.6 \mathrm{~kW}$. The relative increase in hook power was $14,10,9 \%$, respectively, on V, VI and VII gears.

The tractor rolling resistance was determined on the basis of the resistance moment measured during the tests at the entrance to the trackdrive, as the difference between the tangential traction force and the load on the tractor hook.

In the plowed field, it averaged 8.2 and $6.4 \mathrm{kN}$ for the serial and experimental tracks, respectively. A significant reduction in the rolling resistance coefficient from 0.093 to 0.072 occurred due to a reduction in both internal and external losses in the trackdrive as a whole. 
The reduction of internal losses in the trackdrive occurs by reducing the rolling resistance to support rollers on the treadmill of the track. The absence of the front grouser on the experimental track eliminates the main cause of chipping the soil and getting it on the treadmill.

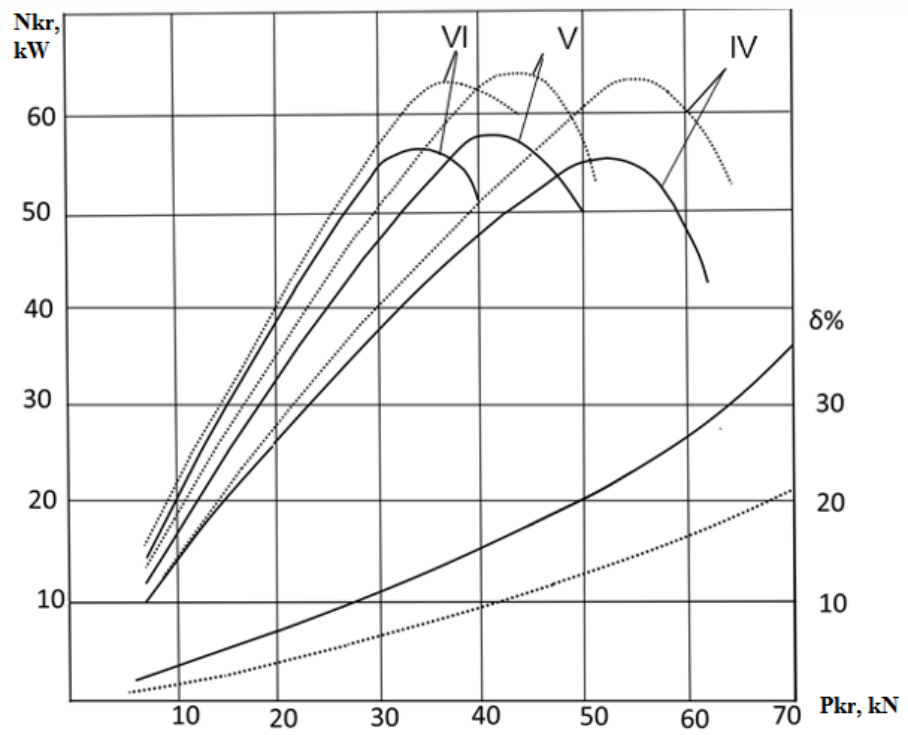

Fig. 8. Traction characteristics of the T-4A tractor on a plowed field. - serial track; - - - experimental track.

The decrease in external losses is explained by a decrease in energy consumption for the introduction of a disjoined grouser into the soil (a smaller area of introduction).

As a result of the conducted studies, epures of angular displacements of adjacent links were obtained at different hook load values.

From the epures of angular displacements (Fig.9), it can be seen that the rotation of adjacent links relative to each other occurs along the entire length of the support surface. At any time, the track chain is a polygonal line in the vertical plane. The maximum value of rotation angle of adjacent links relative to one joint reaches a value of $8 \ldots 14^{\circ}$ in the absence of a load on the hook. This maximum positive value of the angle $\alpha$ is achieved when the joint is positioned in the middle between the guide wheel and the first support roller. The maximum negative value of $\alpha^{\circ}$ in the absence of a hook load is reached along the axis of the last roller.

This fact is explained in the first case, first, by the large center distance of the guide wheel and the first support roller, as well as by the small values of the tensile forces. The maximum value of the negative angle $\alpha$ along the axis of the last roller is mainly due to the inclination angle of the leading branch $\gamma_{2}$ see Figure $4 \mathrm{a}$. The angle of rotation of the adjacent links as they move from the beginning to the end of the support surface decreases slightly due to an increase in the tensile forces in the track contour of the tractor. The total angle of rotation in the joint, which is made by two adjacent tracks, from the moment the link is laid on the support surface until it leaves the soil, is $84^{\circ}$ (plowed field) and $72^{\circ}$ (grain stubble). The value of the total angle $\sum \alpha$ in the tractor T-4 is less than that in the tractor DT-75, which is explained using semi-rigid suspension of the support rollers of the tractor T-4. 
Comparative tests of two track designs, serial and only with rear grouser, on the grain stubble and plowed field, at different loads on the hook indicate that the grouser position does not affect the value of the total angle $\sum \alpha$.

Its maximum value depends on the following:

suspension design of the support rollers;

ratio of the distance between the axes of the support rollers to the step of the track chain link;

values of the tensile forces in the track contour;

physical and mechanical properties of the soil.

The rear grouser eliminates the effect of "scissors", which will reduce the number of erosive-hazardous particles on the trace of the tracks is $30 \ldots 40 \%$, which is $5.6 \ldots 4.25$ times less than in agricultural tractors with a mixed grouser [4].

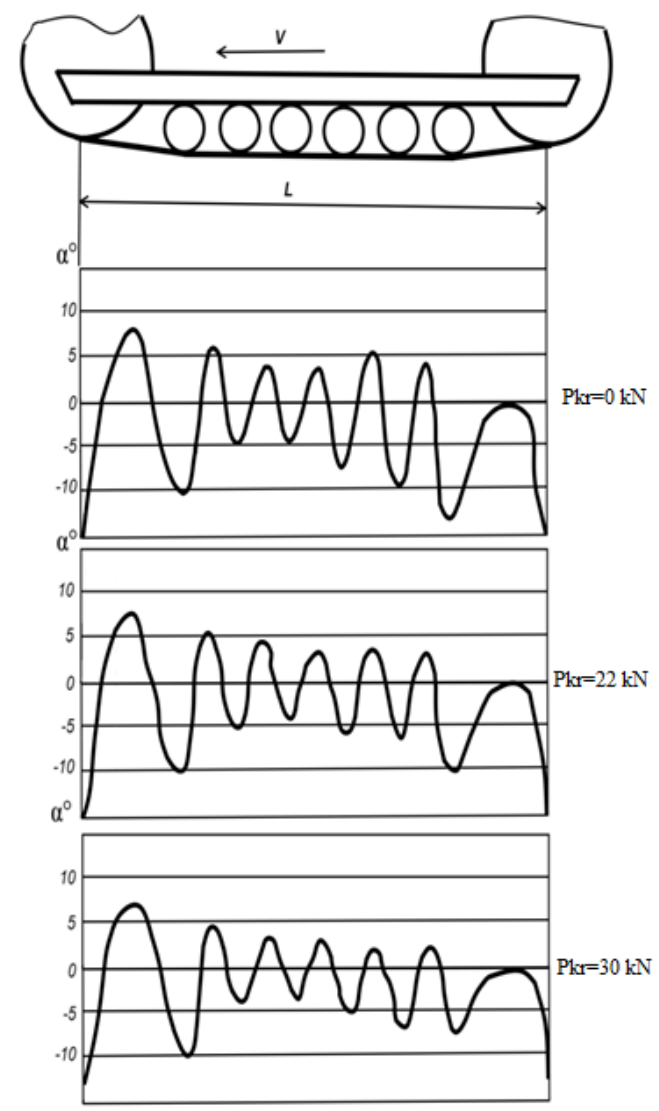

Fig. 9. Epures of relative angular displacements of adjacent links on the support surface tractor T-4A + KPG-2-150

\section{Conclusions}

The traditional design of the metal track link of domestic agricultural tractors with a combined grouser is one of the reasons for increased soil dispersion, its shift along the track trace and incomplete use of the potential traction and coupling capabilities of the trackdrive. A method has been developed for the theoretical evaluation of the influence of the position of the track link on the traction properties of the agricultural tractor, the adequacy of which has been confirmed by experimental studies. The use of a rear grouser 
on the track link will significantly improve the traction properties of the agricultural tractor with the metal track. In this case, when laying a link with a rear grouser, the soil is prepressed in the horizontal direction, which changes the law of distribution of horizontal deformation along the support surface of the trackdrive, which ensures the alignment of the shares of the links in the implementation of the tangential traction force. This leads not only to a reduction in the skidding of the trackdrives, reduction in the rolling losses of the tractor, but also eliminates the "scissors" effect, which will reduce the number of erosionhazardous particles on the track trace and, consequently, reduce the soil destruction. Theoretical studies have shown that the additional horizontal soil deformation from the rotation of the rear grouser in the submerged state significantly depends on its height, the design parameters of the link, as well as the angle of inclination of the lower front branch of the track contour. By changing these parameters, the necessary soil pre-pressing, depending on the external operating conditions of the trackdrive can be obtained. Skidding, at which the maximum tangential traction force develops, for the trackdrive with a front grouser is in the range of $18-20 \%$. In the track with a rear position of the grouserr, due to additional soil pre-pressing, the maximum tangential traction force occurs at lower skidding values by $6 \ldots 9 \%$, which is in good agreement with the results of experimental studies.

Comparative tests of the T-4A tractor with a serial track, and a track on which links the front grousers were removed showed that the maximum traction power increases from 59 to $65 \mathrm{~kW}$, the skidding with a hook load of $40 \mathrm{kN}$ decreases from 14.6 to $9.4 \%$, the rolling resistance coefficient decreases from 0.093 to 0.072 , eliminates the "scissors" effect, which will reduce the number of erosive-dangerous particles in the track trace to $30 \ldots 40 \%$, which is $5.6 . .4 .25$ times less than in agricultural tractors with a mixed grouser, which confirms the effectiveness of using the agricultural tractor with a rear grouser position of the track link.

\section{References}

1. M.A. Rusanov, A.A. Petelin, Proceedings of the International Academy of Agrarian Education 48, 28-33 (2020)

2. V.M. Zabrodsky, A.M. Feinleb, L.N. Kutin, O.L. Utkin-Lyubovtsev, Running systems of tractors: reference book, 271 (Moscow: Agropromizdat, 1986)

3. E.D. Lvov, Theory of the tractor: a textbook for HEIs., 370 (Moscow, Mashgiz, (1946)

4. M.A. Rusanov, V.N. Kozhanov, Bulletin of the International Agrarian Education Academy 45, 58-60 (2019)

5. V.T. Shatalov, Study of the tractor trackdrive $\mathrm{kl} 30 \mathrm{kN}$ interaction with the soil, 20 (Abstract of a thesis ... cand. tech. sciences, Chelyabinsk, 1978)

6. V.A. Skotnikov, A.A. Mashchensky, A.S. Solonsky, Fundamentals of the theory and calculation of the tractor and the car: textbooks and manuals for HEIs, 383 (Moscow: Agropromizdat, 1986)

7. M.A. Rusanov, V.N. Kozhanov, Bulletin of the International Agrarian Education Academy 44, 28-34 (2019)

8. V.V. Katsygin, Minsk: Harvest, 13, 31-64 (1965)

9. M.G. Becker, Introduction to the theory of terrain-machine systems, 520 (Moscow, Mashinostroenie, 1973)

10. V.V. Guskov, A.F. Opeyko, Theory of tracked vehicles rotation, 168 (M, Mashinostroenie, 1984) 
11. I.P. Ksenevich, V.A. Skotnikov, M.I. Lyasko, Running system-soil-harvest, 304 (M, Agropromizdat, 1985)

12. V.N. Naumov, Development of the theory of interaction of the trackdrive with the soil and its implementation when increasing the level of passing ability of transport robots: Abstract of a thesis... doctor of technical sciences: 05.05.03, MSTU n.a. N.E. Bauman. - M., 81 (1993)

13. B.N. Pinigin, Theory of the tractor. Studt of the properties of trackdrives, 92 (Chelyabinsk: ChPI, 1985)

14. Z. Asaf, D. Rubinstein, I. Shmulevich, Journal of Terramechanics 43, 141161 (2006)

15. R. Zhang, J. Li., Journal of Terramechanics 43, 303-316 (2006)

16. A.A. Abyzov, Ensuring the reliability of the elements of the running systems of highspeed tracked vehicles in the design based on the simulation of the operation processes and the formation of failures, 35 (Abstract of a thesis of the doctor of Technical Sciences. - Chelyabinsk, 2014)

17. LS-DYNA user's manual. Version 970 - USA, Livermore CA: Livermore Software Technology Corp., 1564 (2003)

18. V.V. Guskov, N.N. Velev, Yu.E. Atamanov, N.F. Bocharov, I.P. Ksenevich, Tractors. Theory: textbook for university students with spec. "Cars and tractors", 376 (Moscow, Mashinostroenie, 1988) 